\title{
Sports causing most injuries in Hong Kong
}

\author{
K. M. Chan MCh(Orth) FRCS, Yvonne Yuan MSc PCEd, C. K. Li Pg Dip Biomech, P. Chien \\ FRCS and G. Tsang FRCS(Ed) \\ Hong Kong Centre of Sports Medicine and Sports Science, The Chinese University of Hong Kong, Shatin, New \\ Territories, Hong Kong
}

\begin{abstract}
A prospective survey was carried out on 2293 patients attending the Sports Injury Clinic in the Prince of Wales Hospital between May 1984 and December 1990. A Sports Injury Report Form was completed for each patient. Subjects in this study represent a group of nonprofessional and non-élite athletes in a metropolitan area. Soccer, basketball, volleyball, long-distance running and cycling in descending order were the five most common sports causing injury. Different sports produced different injury patterns. In four of the five sports, the knee $(27.27-50.47 \%)$ and the ankle (16.78-24.67\%) were the commonest sites of injury. In cycling, the face (19.46\%) was the commonest site of injury. There was a higher injury rate to the lower than the upper limb in soccer, basketball, volleyball and long-distance running, with a ratio of upper- to lower-limb injury ranging from $1: 1.13$ to 1:46.10. In cycling, upper limb injury was more frequent (upper- to lower-limb injury ratio was 1:0.53). Sprain was the commonest injury overall $(44.60 \%)$. It was also the commonest injury condition in volleyball $(55.15 \%)$, basketball $(55.34 \%)$, soccer $(51.41 \%)$ and long-distance running $(39.33 \%)$. In cycling, abrasion $(24.83 \%)$ was commonest.
\end{abstract}

Keywords: Injury rate, soccer, basketball, volleyball, distance running, cycling

Previous studies have examined injuries in specific sports or in major university and professional athletic programmes $^{1-8}$. This study examines cases treated by a tertiary referral sports injury clinic hospital. Patients in this clinic represent non-élite, non-professional athletes in a metropolitan area.

The purpose of the present study was to investigate the injury pattern in the five sports most commonly associated with injury in Hong Kong. The identification of these could provide a guideline for directing educational resources to the related sports. It is hoped that the present study will also provide guidelines for appropriate planning and staffing for local medical services.

Address for correspondence: Dr K. M. Chan, Hong Kong Centre of Sports Medicine and Sports Science, Chinese University of Hong Kong, Rm 74034, 5/F, Clinical Sciences Building, Prince of Wales Hospital, Shatin, NT, Hong Kong

(C) 1993 Butterworth-Heinemann Ltd

0306 3674/93/040263-05

\section{Subjects and methods}

All patients attending the Sports Injury Clinic in the Prince of Wales Hospital between May 1984 and December 1990 were studied in this investigation. Most of these patients came to the outpatient department of the hospital and claimed that they had been injured in an exercise or sports activity; others were referred to the clinic by the Accident and Emergency Department of the same hospital.

A Sports Injuries Reporting Form was completed for each patient. Information on the form included personal details, diagnosis, sports participation, injury management, and the practice of warm-up and use of protective aids.

\section{Results}

A total of 2293 patients was studied. There were 1537 men and 756 women, $51.59 \%$ were $20-29$ years old, with $95 \%$ in the age range 10-39 years. An almost equal number of amateur, recreational and team players representing league/university/school were included in the study. These athletes comprised $95.86 \%$ of the total. Professional athletes made up only $1.21 \%$. Most of the injured patients had engaged in their sport for over 5 years (969 cases; $42.26 \%$ of the total). Only $10.03 \%$ (230 cases) had engaged in their sport for less than 1 year. The majority of the patients played the sport less than three times per week ( 1810 cases; $78.94 \%$ of the total). More than $60 \%$ of the injured athletes were at the amateur or recreational level. Generally speaking, subjects in the present study had engaged in their sport for many years $(61.72 \%$ for more than 3 years). However, many of them did not practise regularly $-78.94 \%$ practised less than three times per week.

In this study, subjects participated in a total of 48 sports. The five most common sports represented were soccer, basketball, volleyball, long-distance running and cycling (Figure 1). Soccer $(13.91 \%)$ and basketball $(13.43 \%)$ were almost equally important in causing injury.

Injury to the knee ( 805 cases; $35.11 \%$ ) was the most common and injury to the ankle (354 cases; $15.44 \%$ ) the next. Lower-limb injury was more common than upper-limb injury with an injury ratio of $1: 2.98$ (Figure 2). The most common injuries were sprain (1022 cases; $44.60 \%)$ and strain (376 cases; $16.40 \%$ ) (Figure 3). 


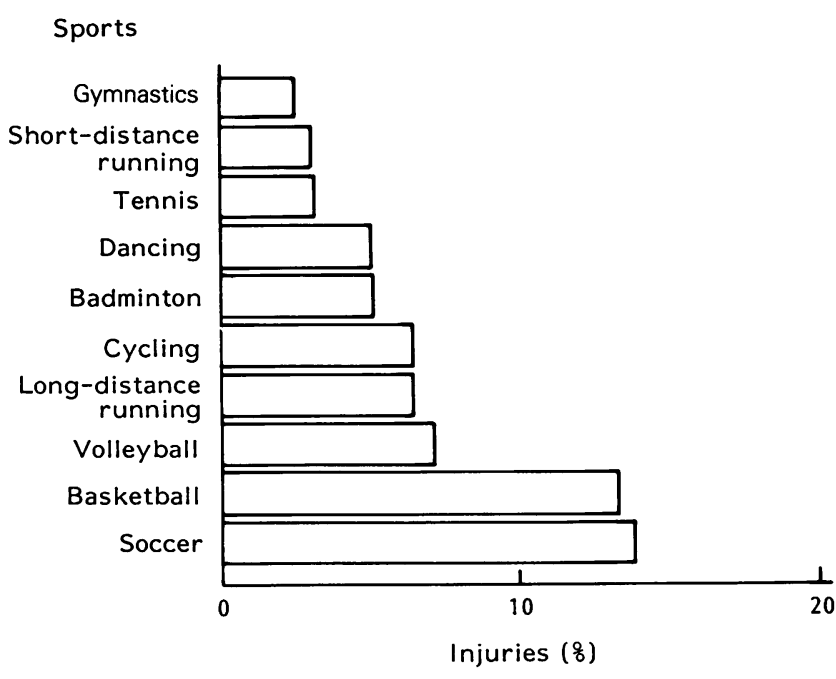

Figure 1. The ten most common sports associated with injury in Hong Kong

In soccer, the sport causing most injuries, the knee ( 161 cases; $50.47 \%$ ) and the ankle ( 55 cases; $17.24 \%$ ) were found to be the most common injury sites. Upper-limb injury made up $7.83 \%$ and lower-limb injury made up $83.07 \%$ of all the injuries. This resulted in an upper- to lower-limb injury ratio of $1: 10.61$. Sprain (164 cases; $51.41 \%$ ) and contusion (59 cases; $18.50 \%$ ) were the most common injuries (Figure 4).

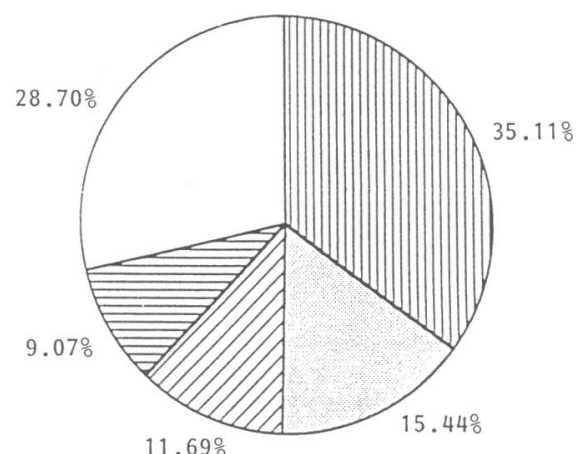

b

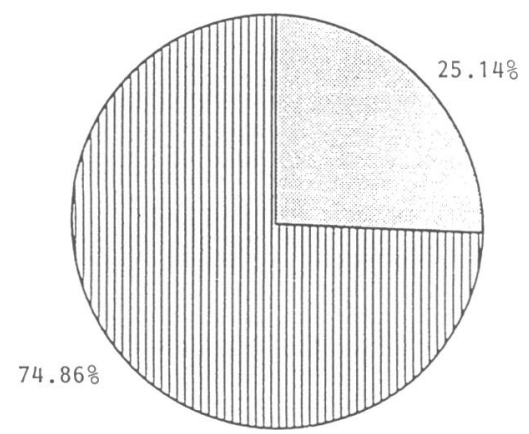

Figure 2. Overall sports related injury - site of injury. a 而 knee; ankle; $\square$ lower spine; 貝 shoulder; $\square$ others. b upper limb; 而 lower limb. $n=2293$
The knee (142 cases; $46.10 \%)$ and the ankle (70 cases; $22.73 \%$ ) were the most frequently injured sites for basketball players. Hand/finger (29 cases; $9.42 \%$ ) and lower spine (23 cases; $7.47 \%$ ) injuries were also common in basketball players. Lower-limb injury $(75.33 \%)$ was more common than upper-limb injury $(16.56 \%)$ (ratio 1:4.55). The most common injury conditions in basketball were sprain (171 cases; $55.52 \%$ ) and overuse (52 cases; $16.88 \%$ ) (Figure 5).

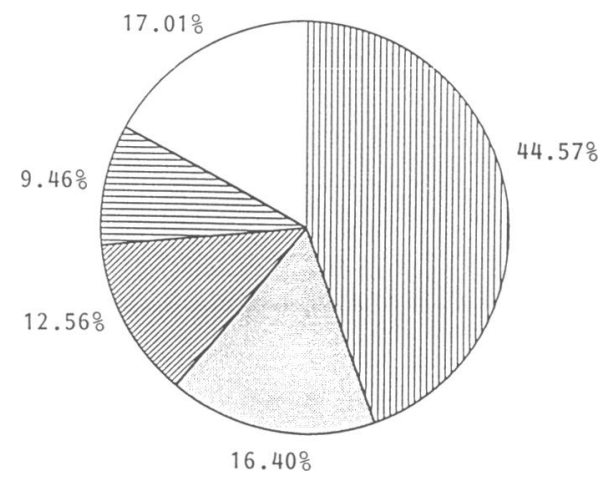

Figure 3. Overall sports related injuries - injury condition, 而 sprain; strain; $\square$ overuse; 自 contusion; $\square$ others. $n=2293$

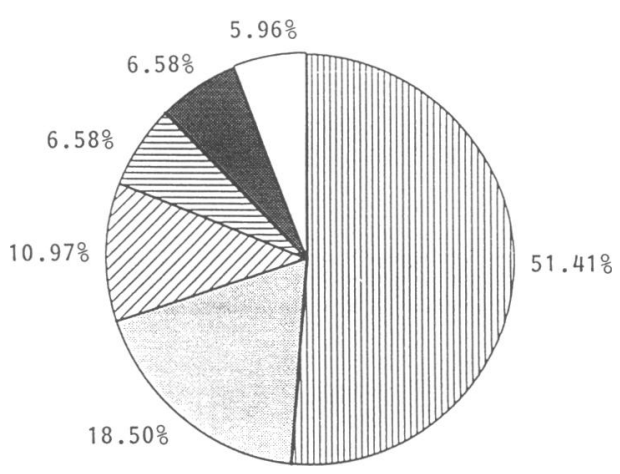

Figure 4. Soccer related injuries - injury condition, 面sprain; $\square$ contusion; $\square$ strain; 目fracture; overuse; others. $n=319$

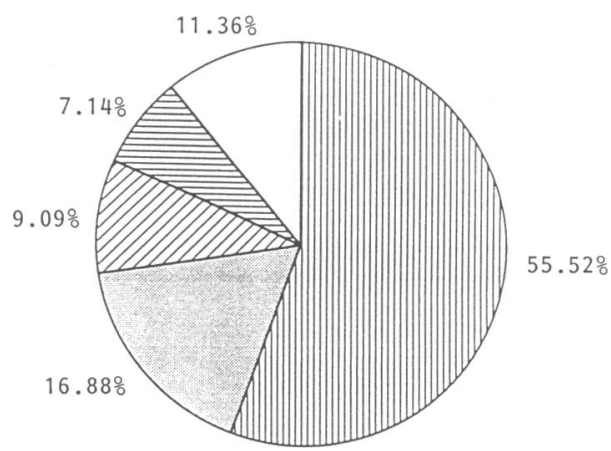

Figure 5. Basketball related injuries - injury condition, 而 sprain; $\square$ overuse; $\square$ contusion; 貝 strain; $\square$ others. $n=308$ 


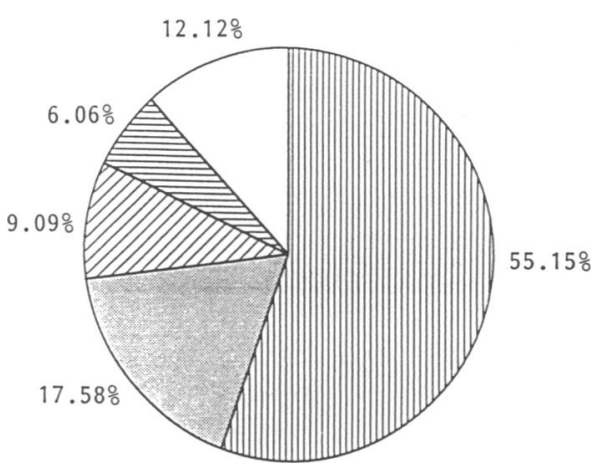

Figure 6. Volleyball related injuries - injury condition, 血 sprain; $\square$ strain; $\square$ overuse; 目 contusion; $\square$ others. $n=165$

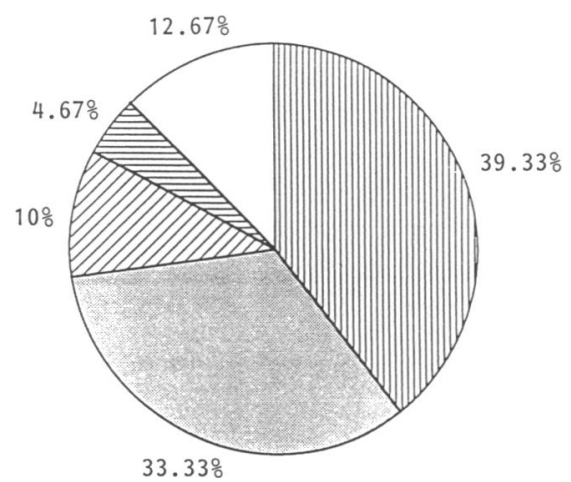

Figure 7. Long-distance running-related injuries - injury

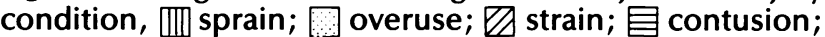
others. $n=150$

a

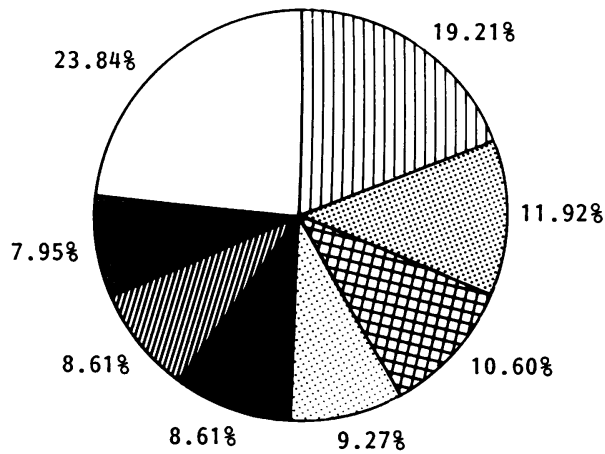

b

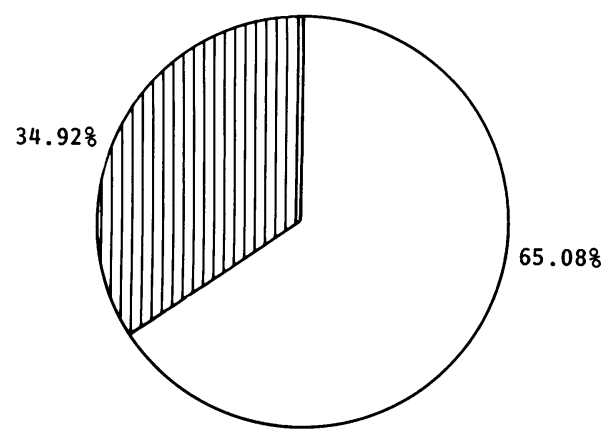

Figure 8. Cycling related injuries - site of injury. a $[\mathbf{l l}$ face; 7 knee; shoulder; 0 forearm; elbow; hand/finger; wrist; $\square$ others. b $\square$ upper limb; 血lower limb. $n=149$

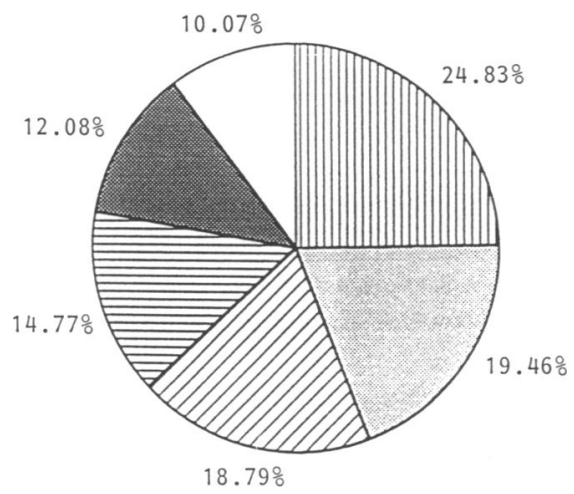

Figure 9. Cycling related injuries - injury condition, 乔 abrasion; laceration; $\square$ fracture; 目 sprain; contusion; $\square$ others. $n=149$

In volleyball, the knee ( 45 cases; $27.27 \%$ ) and the shoulder (39 cases; $23.64 \%$ ) were the most common injury sites. The ankle ( 28 cases; $16.97 \%$ ) and the lower spine ( 21 cases; $12.73 \%$ ) were also common injury sites for volleyball players with an upper- to lower-limb injury ratio of 1:1.13. Sprain (91 cases; $55.15 \%$ ) and strain ( 29 cases; $17.58 \%$ ) were the two most frequent injury conditions found in volleyball related injuries (Figure 6).

Runners were affected most frequently by injury of the knee (75 cases; $50.00 \%$ ) and ankle (37 cases; $24.67 \%$ ). The majority of injuries were to the lower limb $(92.66 \%)$. Upper limb injury only made up $2.01 \%$ of the total (ratio 1:46.10). The most common injury conditions for runners were sprain (59 cases; $39.33 \%$ ) and overuse (50 cases; $33.33 \%$ ) (Figure 7 ).

For cycling, the fifth most common sport represented, the face (35 cases; $23.84 \%$ ) and the knee (28 cases; $19.21 \%$ ) were the most common injury sites (Figure 8). Upper-limb injury $(45.03 \%)$ was more common than lower-limb injury $(24.16 \%)$ in cycling. This resulted in a upper- to lower-limb ratio of 1:0.53. Abrasion (37 cases; $24.83 \%$ ) and laceration (29 cases; $19.46 \%$ ) were the two most frequent injury conditions found in cycling related injuries (Figure 9).

\section{Discussion}

The rate of occurrence of injury was found by this study to be greatest in soccer and basketball. These two sports have been shown to have a high number of injuries by several other studies ${ }^{1-4}$. Although swimming and track and field were found to be associated with a large number of injuries in a study conducted on university students in Hong Kong ${ }^{1}$, these two sports were not included in the top five in this study. This is due possibly to the fact that track and field and swimming are more popular among the student population than the general public in Hong Kong. Football, baseball, field hockey, gymnastics, korfball and handball were also found to be significant injury related sports in previous studies ${ }^{2-4}$ but are not popular among the local population.

The knee was found to be the most common site of injury in this study. Such findings were also well documented in previous studies ${ }^{4-6}$. 
The percentage of knee injury for all the subjects in the study was $35.11 \%$. Athletes in soccer $(50.47 \%)$, long-distance running $(50.00 \%)$ and basketball $(46.10 \%)$ were found to have a higher rate of knee injury than the overall group. A previous study on soccer related injuries ${ }^{6}$ showed that the knee and ankle suffer most from injury. Another study of distance-running related injury ${ }^{7}$ indicated that knee injury was one of the most common injuries incurred through distance running. The findings of the present study were also in agreement with Henry et $a l^{8}$, in which the knee was an important site of injury in basketball players. These sports required a large amount of running and/or sudden turning and stopping. Such requirements put much stress on the knee, resulting in injury. A previous study on cycling related injury ${ }^{9}$ showed that upper limbs were more prone to injury. Similar findings were obtained from the present study in which the forearms and hands of cyclists were more susceptible to injury.

The ankle was found to be the second most common site of injury overall and in long-distance running, basketball and soccer. Its importance is well documented in other studies ${ }^{1,2,4,6-8}$.

The rate of ankle injury for the overall population of athletes was $15.44 \%$. However, a higher ankle injury rate was found in long-distance running $(24.67 \%)$, basketball $(22.73 \%)$, soccer $(17.24 \%)$ and volleyball $(16.78 \%)$. Sports involving more running and jumping seem to result in a higher injury rate to the ankle. The stress imposed on the ankle was thus reflected in the higher injury rate in these sports.

The upper- to lower-limb injury ratio also reflected the stress imposed on the lower limb of athletes in certain sports. Overall, the upper- to lower-limb injury ratio was found to be 1:2.98 in the present study. This ratio is similar to that obtained from the studies of Backx et al. ${ }^{2}$ and Chan et al. ${ }^{1}$

When all the injuries to subjects under study were analysed, the most common injury conditions were ligament sprain (1022 cases; $44.57 \%)$, musculotendinous strain ( 376 cases; $16.40 \%$ ), and overuse microinjury (288 cases; $12.56 \%$ ). The present trend of sports injury type was different from that of the study conducted on university students ${ }^{1}$, in which abrasion, contusion and cramp were the three most common injuries encountered. The difference in the way subjects were recruited in these two studies possibly accounts for the difference in the trend with minor injuries excluded from the present study in which only patients visiting the hospital were enrolled. Backx ${ }^{2}$ studied sports injuries in schoolaged children, and found a predominance of minor injury conditions in which contusion and sprain were the major injuries encountered. A study of sports injuries at the 1985 Junior Olympics ${ }^{3}$ also showed a predominance of minor injuries such as contusion and sprains. Patients who visited the University of Rochester Section of Sports Medicine ${ }^{2}$ had a similar spectrum of injuries to ours.

Sprain was the most frequent injury in four of our top five sports. Soccer, basketball and volleyball demand a lot of sudden turning and jumping while long-distance running tends to cause sprain when done on an uneven surface. The present finding is in agreement with Ekstrand and Gillquist ${ }^{6}$, who found sprain to be the most frequent injury in soccer players with contusion and strain also common.

Microinjuries from overuse were common in long-distance running $(33.33 \%)$ and basketball $(16.88 \%)$. Running involves repeated movement and tends to cause microscopic injuries to the lower limb. A study of all participants in a popular $16-\mathrm{km} \mathrm{race}^{7}$ indicated that over $70 \%$ of running-related injuries were due to stress and overuse. This much higher percentage of overuse injury in a population of runners is most likely due to the high training volume required in preparation for a race.

Cyclists incurred different injuries from those of other athletes. Abrasion (24.83\%), laceration $(19.46 \%)$ and fracture $(18.79 \%)$ were most common and resulted mostly from accidents. Tucci and Barone $e^{9}$ found that contusion $(27.3 \%)$, fracture $(20 \%)$ and laceration $(18.6 \%)$ were most common for cyclists. Furthermore, abrasion and contusion were also common in a study on a population of university student cyclists ${ }^{1}$.

The top five sports represented in this injury study were soccer, basketball, volleyball, long-distance running and cycling. This does not show that these sports are more risky than other sports but that these sports do lead to a significant number of sports injuries.

Overall, the knee and ankle were the most common sites of injury. In general, weight-bearing sports that required a lot of running, turning and jumping (soccer, basketball, volleyball, long-distance running) led to more injury to the knee and ankle. Non-weight-bearing sports such as cycling were found to be less important in causing lower limb injury.

Sprain was the most common injury seen. Sports that demand a high degree of sudden turns and forceful jumping (soccer, basketball, volleyball), were found to be particularly important in causing sprain. A similar trend was not found in sports such as cycling that involve repetitive movements. Although long-distance running also involves repetitive movements, running on uneven surfaces may also lead to a greater rate of sprains.

The findings in the present study indicate that different sports caused different injury patterns in terms of both injury site and injury condition.

This study has paved the way for further research in the following directions: (1) epidemiological study of sports injury in specific age and sports groups; (2) surveillance of sports-specific injuries; (3) delineation of preventive measures in the light of these epidemiological findings.

\section{References}

1 Chan KM, Fu F, Leung L. Sports injuries survey on university students in Hong Kong. Br J Sports Med 1984; 18: 195-202.

2 Backx FJG, Erich WBM, Kemper ABA, Verbeek ALM. Sports injuries in school-aged children - an epidemiological study. Am J Sports Med 1989; 17: 234-9.

3 Martin RK, Yesalis CE, Foster D, Albright JP. Sports injuries at the 1985 Junior Olympics - an epidemiologic analysis. Am J Sports Med 1987; 15: 603-8. 
4 DeHaven KE, Lintner DM. Athletic injuries: comparison by age, sport, and gender. Am J Sports Med 1986; 14: 218-24.

5 Kannus $\mathrm{P}$, Aho $\mathrm{H}$, Jarvinen M, Niittymaki S. Computerized recording of visits to an outpatient sports clinic. Am J Sports Med 1987; 15: 79-85.

6 Ekstrand J, Gillquist J. Soccer injuries and their mechanisms: a prospective study. Med Sci Sports Exerc 1983; 15: 267-70.
7 Marti B, Vader JP, Minder CE, Abelin T. On the epidemiology of running injuries - the 1984 Bern Grand-Prix study. Am J Sports Med 1988; 16: 285-94.

8 Henry JH, Laureau B, Neigut D. The injury rate in professional basketball. Am J Sports Med 1982; 10: 16-18.

9 Tucci JJ, Barone JB. A study of urban bicycling accidents. Am J Sports Med 1988; 16: 181-4.

\title{
The Physiology and PATHOPhysiology of EXERCISE TOLERANCE
}

\author{
ULM, GERMANY
}

\section{1-24 SEPTEMBER 1994}

This meeting is designed to consider exercise intolerance in both clinical and sporting contexts. Bringing together scientists, with a wide range of expertise, for critical consideration of these issues should improve our understanding of both exercise limitation and methods of improving exercise tolerance.

The programme consists of invited lectures by internationally-recognised experts and also poster presentations and discussions.

Topics will include:

- The physiological basis of muscular fatigue

- Systemic limitations to maximum exercise in healthy subjects

- $\quad$ Pathophysiology of exercise intolerance

- $\quad$ Sports-specific limitations to exercise in health and disease

- $\quad$ Enhancing exercise tolerance in health and disease.

For further information, please contact: Department of Sports Medicine, Organizing Committee, 89070 Ulm, Germany. Tel: +49 7315026961 . Fax: +49 7315026686. 